\title{
Restricted Repetitive Sampling in Designing of Control Charts
}

\author{
Muhammad Anwar Mughal \\ Department of Statistics \\ National College of Business Administration \& Economics, Pakistan \\ formanteacher@gmail.com \\ Muhammad Azam \\ Department of Statistics and Computer Science \\ University of Veterinary and Animal Sciences, Pakistan \\ mazam72@yahoo.com \\ Muhammad Aslam \\ Department of Statistics, Faculty of Sciences \\ King Abdulaziz University, Jeddah 21551, Saudi Arabia \\ aslam_ravian@hotmail.com
}

\begin{abstract}
In this article a criteria have been defined to classify the existing repetitive sampling into soft, moderate and strict conditions. Behind this division a ratio has been suggested i.e. $c_{2}$ (constant used in repetitive limits) to $c_{1}$ (constant used in control limit) in slabs. A restricted criterion has been devised on the existing repetitive sampling. By embedding the proposed schematic in the control chart it becomes highly efficient in detecting the shifts quite earlier as well as it detects even smaller shifts at smaller ARLs. To facilitate the user for best choice the restricted criterion has further categorized to softly restricted, moderately restricted and strictly restricted. The restricted conditions are dependent on value of restriction parameter 'm' varies 2 to 6. The application of proposed scheme on selected cases is given in tables which are self-explanatory.
\end{abstract}

Keywords: ARL, Repetitive sampling, Shift, Alarm, Control limits, Process control.

\section{Introduction}

Statistical process control tools are eyes of quality assurance department in any organization. The vision of these eyes is mainly dependent on the efficiency of tools and techniques used under the process control monitoring. In SPC, control charts have a vital role among the techniques. In the beginning the basic charts were not that smart to alarm at medium and small shifts. The Quality Control Statisticians and experts have been working to improve out-of-control detections by introducing several chart types, sampling techniques and merger of techniques etc. Initially, simple random sampling was the technique used to pick a subgroup for charting purpose. With the passage of time a lot of developments were taken place in the subject of process control and other sampling techniques were introduced. These techniques improved the performance of control chart. In the present literature a technique Repetitive sampling is frequently found in the quality control tools. This technique was introduced in accepting sampling by (Sherman, 1965) and still found in sampling plans literature. In the material reviewed related to sampling plans in last couple of years (Balamurali, Aslam, \& Jun, 2015) introduced a sampling plan using skip-lot sampling by re-sampling technique. (Wu, Aslam, Chen, \& Jun, 2015) developed a group acceptance sampling plan using repetitive sampling. (Aslam, Jun, Fernández, Ahmad, \& Rasool, 2014) proposed a group sampling plan on truncated Tests 
by using repetitive sampling method. (Yen, Chang, \& Aslam, 2015) presented variable acceptance sampling plan for one sided specification based on repetitive sampling. Later, this technique crept in control chart theory and construction. Such as(Lee, Aslam, Shakeel, Lee, \& Jun, 2015) develop a control chart to monitor process mean by using auxiliary information. (Ahmad, Aslam, \& Jun, 2014a) proposed a control chart for Coal Quality monitoring. (Aslam, Azam, \& Jun, 2014b) proposed new charts for both attribute and variables. (Ahmad, Aslam, \& Jun, 2014b) designed x-bar chart by using process capability. (Azam, Aslam, \& Jun, 2015) designed a control chart and showed improvements. (Aslam, Ahmad, \& Osama, 2015) Introduced a control chart for attribute data and from the same author (Aslam, Azam, \& Jun, 2014a) developed a non-parametric type mixed chart based. (Aslam, Khan, Azam, \& Jun, 2014) design a t-chart using same sampling technique. (Aslam, Azam, \& Jun, 2016)present a control chart on COMPoisson distribution with re-sampling. (Aslam, Azam, Khan, \& Jun, 2015) applied repetitive sampling on a mixed chart. From the same author in (Aslam, Khan, \& Azam, 2016) x-bar control chart for non-normal correlated data was proposed. In all the above mentioned cases repetitive sampling technique was applied.

In sampling plans, the subgroups are selected to take a decision about a lot for its rejection or acceptance whereas in control charts a subgroup selected is used to monitor the process variation when the process is intact or process is in flow. The outcome of the selected subgroup and the pattern behavior of earlier subgroups direct the quality supervisor to leave the process with current settings, to defer the subgroup and make adjustments in the process or to hunt special cause of variation. In the situation when many samples are deferred as un-plotted, they address a change in the process settings. It indicates instability in the process or in other words an insertion of assignable cause of variation. This point is basically a ground for purposed controlled chart procedure. The proposed scheme is about to cap the number of deferred subgroups in repetitive sampling. In repetitive sampling based control charts since there are two additional limits say repetitive limits inside the usual control limits (UCL \& LCL), these limits found in literature being located between 2-sigma limits and extreme limits (3-sigma) or even in many cases they are located inside 1-sigma limits in the discussed charts. These control charts defines a very strict criteria for monitoring process shifts that perhaps leave no stone unturned. The strictness of the criteria depends on the location of repetitive limits. As repetitive limits are close to (CL) stricter is the criteria to capture shifts. In the literature the most popular yard stick used to gage the performance of a chart is ARLs. It facilitates the reader to gage and compare different techniques. Based on the ARLs of repetitive control charts, repetitive sampling found very effective yet we are unable to determine the constraints, prose and cones lying underway in their application. To categorize these charts as Soft, Moderate or Strict the authors have obtained an index as a ratio of repetitive limits constant $\left(c_{2}\right)$ to control limits constant $\left(c_{1}\right)$ i.e. $c_{2} / c_{1}$. To interpret this index if the ratio for example is 0.90 indicates that constant c2is 0.90 times of constant $c_{1}$. In this situation, control limits and repetitive limits are very close to each other as such, the likelihood of subgroups to fall between them is very little, therefore such control chart can be considered as one with marginally strict (soft). Explaining in a bit different way the repetitive limits in above situation are located between warning limits $( \pm 2 / 3$ S.E.) and control limits (extreme limits). In a similar vice versa conditions when the ratio is below 0.33 , the repetitive limits are located between CL and ( $\pm 1 / 3$ S.E.) limits. This can be assumed as an example of strict repetitive-based control chart. Here, 
the repetitive limits are far away from extreme limits, cause a wider repetitive zone with higher likelihood of subgroups falling in it. Thus, the authors categorize repetitive scheme as very strict if ratio $\left(c_{2} / c_{1}\right)$ is less than 0.33 , slightly strict if ratio is 0.67 or higher and moderately strict otherwise. It suggests that these charts need to apply in the situation where the quality parameters and specs in the certain product are highly crucial. Often in highly sophisticated product or when specs are highly precise, the control chart of such processes displays well in-control condition but specs are being compromised. Due to this factor some lots are sold to secondary customers at low pricing or even sometime product is scrapped. The best use of repetitive based control charts can be the situation as mentioned above. Now discussing the literature that has been reviewed particularly with reference to above mentioned ratio so that work done so far can be interpreted in similar terms. In the related reviewed literature(Azam et al., 2015)used the constants in charts their ratio $\left(c_{2} / c_{1}\right)$ remain 0.33 these may be considered as moderately strict condition, In (Aslam, Ahmad, et al., 2015)used soft repetitive conditions as the ratio in different proposals found 0.56 to 0.79, (Aslam, Azam, et al., 2014a)applied different charts with ratio 0.33 to 0.40 these repetitive conditions are moderate. In other articles quite smaller ratios were used in repetitive based charts as (Aslam, Khan, et al., 2014)used 0.26 it indicates strict conditions, (Aslam, Azam, et al., 2016) further lower the ratio to 0.15 and 0.16. In a mixed control chart by (Aslam, Azam, et al., 2015)different ratios found as $0.27,0.011,0.19,0.08,0.25,0.22$ and 0.04 these two articles are based on extremely strict repetitive conditions for example when ration is 0.011, rarely the subgroups will likely to fall in in-control region. In (Aslam, Khan, et al., 2016) the suggested $x$-bar chart for a correlated data different ratios were used that vary from 0.07 to 0.89 in this article all conditions i.e. lenient to strict have been applied. Other than above mentioned articles sufficient material is available where in almost cases very strict repetitive ratios have been observed such as (Aslam, Azam, et al., 2014b) and (Ahmad et al., 2014b).

In the above review we find smallest ratios as $0.011,0.04$ and 0.07 , these indicate very strict control charts where it is obvious that due to very large repetitive zone, large number of subgroups might be deferred if these charts are applied to any process. Such charts can be useful when very high precision is required in the product and a slight departure in quality characteristics is crucial for process owner.

\section{Mechanism and Construction of the Proposed Scheme}

Restricted repetitive sampling scheme is quite similar to repetitive sampling but it restricts the number of subgroups falling in repetitive zone. The proposed criteria suggests that deferred number of subgroups should be capped to $(\mathrm{i} / \mathrm{m})$ times of total number of subgroups drawn for control chart monitoring ( $\mathrm{m}$ being restriction parameter). In the usual repetitive sampling based control charts the process is declared as in-contact if subgroup falls between upper and lower repetitive limits and out of control if it falls outside extreme limits if the subgroup falls otherwise it is replaced with another drawing unless above two conditions exist. The proposed criteria suggests that if number of deferred subgroups becomes larger than $(\mathrm{i} / \mathrm{m})$, the process be declared as out of control as it does not make sense to bear a sequence of deferred subgroups in control chart. Here ' $i$ ' is total number of subgroups drawn and ' $\mathrm{m}$ ' is restriction parameter varies that 2 to 6 on the basis of repetitive strictness category described earlier. For instance if $i=10$ and 
selected $m=2$, the restricted repetitive scheme allows maximum deferred samples as 5 . Falling of another subgroup in repetitive zone process will be declared as out-of-control. When $\mathrm{m}=2$ the proposed criteria is most lenient and at $\mathrm{m}=6$ it is the most strict.

The authors categorized the scheme as slightly restricted when $\mathrm{m}=2$, moderately restricted as $m=3$ or 4 and highly restricted when $m=5$ or 6 . The categorization of existing repetitive sampling has been associated with ratios and suggested restriction parameters are given in the table below.

\section{Table 1:}

\begin{tabular}{lll}
\hline Repetitive condition & Ratio(c2/c1) & Proposed ' $^{\text {' }}$ \\
\hline Strict & $\leq 0.33$ & 2 or 3 \\
Slightly strict & $\geq 0.67$ & 2 to 6 \\
Moderate & Otherwise & 2 to 5 \\
\hline
\end{tabular}

By embedding the proposed criteria in repetitive sampling the control charts become more efficient in alarming at relatively smaller shifts, otherwise monitoring is quite safe from any false alarm.

\section{Design of control chart and Monte Carlo algorithm}

Let a sample of size $\mathrm{n}$ from a process follow $\mathrm{N}\left(\mu, \sigma^{2}\right)$. When the process is in control at $\mu_{0}$ the control limits and repetitive limits are calculated as:

$$
\begin{aligned}
& U C L=\mu_{0}+c_{1} \sigma / \sqrt{n} \\
& U R L=\mu_{0}+c_{2} \sigma / \sqrt{n} \\
& C L=\mu_{0} \\
& L R L=\mu_{0}-c_{2} \sigma / \sqrt{n} \\
& L C L=\mu_{0}-c_{1} \sigma / \sqrt{n}
\end{aligned}
$$

The working above control chart is as follows

Step1. Selecting a random sample of size $\mathrm{n}$ from the process and computing estimator mean as $\bar{x}=\frac{\sum x}{n}$

Step2. The process is in control if $L R L<\bar{x}<U R L$

Step3. The process is out of control if $\bar{x}>U C L$ or $\bar{x}<L C L$

If $L C L<\bar{x} \leq L R L$ or $U R L \leq \bar{x}<U C L$, then go to Step1 provided that number of subgroups satisfying given conditions are less or equal to ' $\mathrm{k}$ '. Otherwise process is out of control.

Here $\mathrm{k}=\mathrm{i} / \mathrm{m}$

Algorithm: Monte Carlo Simulation Program of Control Chart for in-control and shifted process 
Following are algorithmic steps involved in Monte Carlo Simulation R program.

(1) Generate a random sample of size $\mathrm{n}$ from the Normal Distribution with associated parameters for in-control process. Generate 100,000 subgroups.

(1.1) Obtaining statistic $\bar{x}$ from each subgroup

(2) Setting up control limits

(2.1) Selecting suitable values of ' $\mathrm{m}$ ' and $\mathrm{c}_{1} / \mathrm{c}_{2}$ ratio

(2.2) Compute UCL and LCL by using a specific constant $c_{1}$ in the relevant formula

(2.3) Compute URL and LRL by using a specific constant $c_{2}$ in the relevant formula

(2.4) Keeping in view the working procedures of the control chart, recording the subgroup's number as run length at which the process is declared as out of control.

(2.5) Repeat above Step a sufficient number of times (say 10,000) to calculate the in-control ARL. If the in-control ARL is equal to the desired ARL 0 , then go to Step 3 with the selected value of $c_{1}$ and $c_{2}$. Otherwise, modify the value of constant $c_{1}$ and repeat Steps 2.2 to 2.5

(3) Evaluating the out-of-control ARL

(3.1) Generate a random sample of size $\mathrm{n}$ from the Normal Distribution with associated parameters for shifted process. Generate 100,000 such subgroups.

(3.2) Compute $\bar{x}$ statistic for each sample

(3.3) Applying working criteria of the chart on the obtained 100,000 values of $\bar{x}$

(3.4) Repeat Steps 3.1 to 3.3 until the process is declared as out-of-control. Record the number of subgroup as a run length that show out of control signal.

(4) Repeat all the above mentioned steps 10,000 times to obtain the Average Run Length.

\section{Performance of Control Chart at Different Settings}

In the following tables (2 to 6) ARLs have been computed by using Monte Carlo simulation procedure to observe the efficiency of the chart at different repetitive conditions and applied restrictions. In all the tables below where $\mathrm{m}=1$ indicates results based on existing repetitive sampling. At $m=2$, results are obtained where soft restricted condition applied, at $m=3$ or 4 can be said as moderately restricted and at $m=5$ or 6 the restricted conditions are strict. In Table 2 and 3, restriction criteria have been experimented on lenient repetitive condition where $c_{2} / c_{1}$ ratio is equal to 0.75 . ARLs have been computed at sample sizes 5, 10, 20 and 40 by applying all discussed restricted conditions (light, moderate, strict). Table 2 and 3 show $\mathrm{ARL}_{1} \mathrm{~s}$ when $\mathrm{ARL}_{0}$ are respectively 500 and 370 . It is very clear from the results that as the restriction parameter increases the efficiency of the monitoring improves. The sample size does have impact on 
the results, its significance can also be observed from the tables. Similarly, in Table 4 and 5 , restriction criteria have been experimented on lenient repetitive condition where $\mathrm{c} 2 / \mathrm{c} 1$ ratio is equal to 0.67 . ARLs have been computed at sample sizes 5, 10, 20 and 40 by applying all discussed restricted conditions (light, moderate, strict). Table 4 and 5 show $\mathrm{ARL}_{1} \mathrm{~s}$ when $\mathrm{ARL}_{0}$ are respectively 500 and 370. In Table 6 restriction criteria have been applied to moderate repetitive conditions where ratio is 0.50 . ARLs have been computed at sample sizes 5, 10, 20 and 40 with $\mathrm{ARL}_{0}=370$. It is suggested that under moderate repetitive conditions restriction parameter should kept below 6 . In case it is desired to apply $\mathrm{m}=6$, this may require a reduction in $\mathrm{ARL}_{0}$. In Table 7 restriction criteria have been experimented on strict repetitive conditions where ratio is 0.33 . In case of strict repetitive conditions the likelihood of subgroups falling in repetitive zone is very high as such the chart is already very smart in detecting smaller shifts. In this situation the monitoring can be made stricter even at soft restrictions. To describe the findings more widely Table 2 to 6 have been discussed in Table 7 in detail.

Table 2: $\quad c_{2}=0.75 c_{1}, A R L_{0}=500$

\begin{tabular}{|c|c|c|c|c|c|c|}
\hline & \multicolumn{6}{|c|}{$n=5$} \\
\hline shift & $m=1$ & $\mathrm{~m}=\mathbf{2}$ & $\mathbf{m}=\mathbf{3}$ & $\mathrm{m}=4$ & $m=5$ & $m=6$ \\
\hline 0.00 & 496.95 & 507.38 & 494.34 & 503.18 & 494.57 & 505.96 \\
\hline 0.10 & 410.31 & 395.93 & 404.59 & 403.65 & 418.28 & 426.59 \\
\hline 0.15 & 318.06 & 310.55 & 316.42 & 319.97 & 327.98 & 322.59 \\
\hline 0.20 & 229.78 & 234.99 & 235.95 & 241.09 & 249.56 & 228.34 \\
\hline 0.25 & 169.89 & 178.53 & 168.64 & 163.55 & 170.79 & 163.90 \\
\hline 0.30 & 133.08 & 127.59 & 127.59 & 119.97 & 122.36 & 116.39 \\
\hline 0.35 & 95.20 & 98.04 & 85.00 & 88.78 & 79.61 & 76.75 \\
\hline 0.40 & 72.03 & 70.66 & 64.33 & 61.36 & 58.37 & 54.54 \\
\hline 0.45 & 51.39 & 51.24 & 44.58 & 42.89 & 39.81 & 36.87 \\
\hline 0.50 & 38.53 & 37.85 & 34.06 & 30.15 & 29.36 & 25.97 \\
\hline 0.60 & 23.28 & 21.46 & 17.41 & 14.95 & 13.25 & 11.30 \\
\hline 0.70 & 13.33 & 12.67 & 9.65 & 7.87 & 7.00 & 6.01 \\
\hline $\mathbf{0 . 8 0}$ & 8.76 & 7.62 & 5.10 & 4.46 & 3.73 & 3.34 \\
\hline 0.90 & 5.66 & 4.59 & 3.27 & 2.85 & 2.64 & 2.48 \\
\hline \multirow[t]{2}{*}{1.00} & 3.90 & 3.06 & 2.14 & 1.99 & 1.93 & 1.84 \\
\hline & \multicolumn{6}{|c|}{$n=10$} \\
\hline 0.00 & 511.35 & 502.16 & 492.48 & 497.57 & 513.86 & 492.26 \\
\hline 0.10 & 331.68 & 338.61 & 328.96 & 332.55 & 343.96 & 342.81 \\
\hline 0.15 & 225.35 & 224.98 & 214.40 & 215.94 & 223.13 & 206.72 \\
\hline 0.20 & 142.03 & 141.98 & 138.32 & 137.35 & 135.05 & 131.82 \\
\hline 0.25 & 91.66 & 91.24 & 84.58 & 83.05 & 80.60 & 76.80 \\
\hline 0.30 & 59.61 & 59.76 & 54.13 & 51.10 & 49.58 & 45.38 \\
\hline 0.35 & 39.49 & 40.12 & 33.34 & 31.53 & 28.84 & 26.21 \\
\hline 0.40 & 27.81 & 26.29 & 21.98 & 17.89 & 16.89 & 14.60 \\
\hline 0.45 & 18.70 & 17.63 & 13.50 & 11.37 & 11.08 & 9.14 \\
\hline 0.50 & 13.73 & 11.77 & 8.95 & 7.38 & 6.21 & 5.50 \\
\hline 0.60 & 6.98 & 5.96 & 4.16 & 3.53 & 3.10 & 3.02 \\
\hline 0.70 & 4.04 & 3.24 & 2.28 & 2.02 & 2.01 & 1.89 \\
\hline 0.80 & 2.52 & 1.97 & 1.56 & 1.51 & 1.48 & 1.50 \\
\hline 0.90 & 1.80 & 1.49 & 1.27 & 1.24 & 1.22 & 1.26 \\
\hline 1.00 & 1.39 & 1.25 & 1.13 & 1.10 & 1.12 & 1.10 \\
\hline
\end{tabular}


Restricted Repetitive Sampling in Designing of Control Charts

\begin{tabular}{|c|c|c|c|c|c|c|}
\hline & \multicolumn{6}{|c|}{$n=20$} \\
\hline 0.00 & 511.19 & 496.35 & 506.23 & 501.14 & 511.69 & 507.83 \\
\hline 0.10 & 229.94 & 240.78 & 230.20 & 231.04 & 234.18 & 226.20 \\
\hline 0.15 & 126.54 & 131.78 & 123.51 & 120.49 & 120.53 & 114.44 \\
\hline 0.20 & 68.76 & 68.18 & 61.81 & 59.99 & 60.03 & 52.87 \\
\hline 0.25 & 37.97 & 39.53 & 33.10 & 29.40 & 28.79 & 24.40 \\
\hline 0.30 & 22.76 & 21.54 & 17.14 & 15.13 & 13.92 & 11.94 \\
\hline 0.35 & 13.71 & 12.48 & 9.81 & 7.84 & 6.76 & 5.85 \\
\hline 0.40 & 8.71 & 7.36 & 5.28 & 4.28 & 3.78 & 3.48 \\
\hline 0.45 & 5.80 & 4.76 & 3.04 & 2.79 & 2.71 & 2.42 \\
\hline 0.50 & 3.84 & 3.18 & 2.23 & 1.98 & 1.89 & 1.88 \\
\hline 0.60 & 2.10 & 1.76 & 1.37 & 1.33 & 1.33 & 1.31 \\
\hline 0.70 & 1.46 & 1.26 & 1.15 & 1.13 & 1.14 & 1.14 \\
\hline 0.80 & 1.14 & 1.09 & 1.06 & 1.05 & 1.05 & 1.04 \\
\hline 0.90 & 1.06 & 1.03 & 1.02 & 1.02 & 1.02 & 1.03 \\
\hline \multirow[t]{3}{*}{1.00} & 1.01 & 1.02 & 1.01 & 1.01 & 1.02 & 1.01 \\
\hline & \multicolumn{6}{|c|}{$\mathbf{n}=\mathbf{3 0}$} \\
\hline & $m=1$ & $\mathbf{m}=\mathbf{2}$ & $\mathbf{m}=\mathbf{3}$ & $\mathrm{m}=4$ & $m=5$ & $m=6$ \\
\hline 0.00 & 491.58 & 510.06 & 486.26 & 499.09 & 512.67 & 501.35 \\
\hline 0.10 & 181.09 & 180.11 & 179.19 & 172.32 & 175.71 & 172.17 \\
\hline 0.15 & 86.07 & 84.79 & 78.79 & 74.94 & 74.20 & 71.31 \\
\hline 0.20 & 42.01 & 40.55 & 35.51 & 32.42 & 30.70 & 27.41 \\
\hline 0.25 & 22.12 & 20.18 & 16.28 & 13.66 & 12.78 & 10.49 \\
\hline 0.30 & 12.00 & 10.47 & 7.80 & 6.09 & 5.23 & 4.73 \\
\hline 0.35 & 6.71 & 5.64 & 3.90 & 3.31 & 3.03 & 2.81 \\
\hline 0.40 & 3.96 & 3.50 & 2.35 & 2.09 & 1.97 & 1.94 \\
\hline 0.45 & 2.72 & 2.20 & 1.65 & 1.58 & 1.55 & 1.55 \\
\hline 0.50 & 1.93 & 1.68 & 1.31 & 1.35 & 1.30 & 1.29 \\
\hline 0.60 & 1.32 & 1.19 & 1.09 & 1.09 & 1.08 & 1.08 \\
\hline 0.70 & 1.10 & 1.05 & 1.03 & 1.03 & 1.03 & 1.03 \\
\hline 0.80 & 1.02 & 1.02 & 1.01 & 1.02 & 1.01 & 1.01 \\
\hline 0.90 & 1.00 & 1.00 & 1.01 & 1.00 & 1.00 & 1.00 \\
\hline \multirow[t]{2}{*}{1.00} & 1.00 & 1.00 & 1.00 & 1.00 & 1.00 & 1.00 \\
\hline & \multicolumn{6}{|c|}{$\mathrm{n}=\mathbf{4 0}$} \\
\hline 0.00 & 506.04 & 482.52 & 498.83 & 502.54 & 512.64 & 500.82 \\
\hline 0.10 & 147.17 & 141.56 & 137.90 & 132.36 & 136.04 & 126.97 \\
\hline 0.15 & 58.15 & 59.04 & 53.62 & 51.17 & 49.25 & 44.91 \\
\hline 0.20 & 27.80 & 27.31 & 21.27 & 19.13 & 17.03 & 14.95 \\
\hline 0.25 & 13.03 & 12.48 & 9.03 & 7.26 & 6.26 & 5.78 \\
\hline 0.30 & 6.98 & 6.12 & 4.05 & 3.40 & 3.30 & 3.00 \\
\hline 0.35 & 3.90 & 3.28 & 2.26 & 1.98 & 2.01 & 1.95 \\
\hline 0.40 & 2.52 & 2.05 & 1.56 & 1.49 & 1.46 & 1.49 \\
\hline 0.45 & 1.76 & 1.50 & 1.26 & 1.28 & 1.25 & 1.24 \\
\hline 0.50 & 1.40 & 1.25 & 1.12 & 1.10 & 1.11 & 1.11 \\
\hline 0.60 & 1.10 & 1.05 & 1.02 & 1.04 & 1.04 & 1.02 \\
\hline 0.70 & 1.02 & 1.01 & 1.01 & 1.01 & 1.01 & 1.01 \\
\hline 0.80 & 1.00 & 1.00 & 1.00 & 1.00 & 1.00 & 1.00 \\
\hline 0.90 & 1.00 & 1.00 & 1.00 & 1.00 & 1.00 & 1.00 \\
\hline 1.00 & 1.00 & 1.00 & 1.00 & 1.00 & 1.00 & 1.00 \\
\hline
\end{tabular}


Table 3: $\quad c_{2}=0.75 c_{1}, A_{R L}=370$

\begin{tabular}{|c|c|c|c|c|c|c|}
\hline & \multicolumn{6}{|c|}{$n=5$} \\
\hline Shift & $m=1$ & $\mathrm{~m}=2$ & $\mathbf{m}=\mathbf{3}$ & $\mathrm{m}=4$ & $m=5$ & $m=6$ \\
\hline 0.00 & 367.23 & 367.57 & 359.07 & 368.11 & 375.27 & 383.33 \\
\hline 0.10 & 305.56 & 292.07 & 294.01 & 286.57 & 290.70 & 306.02 \\
\hline 0.15 & 239.80 & 226.93 & 232.60 & 232.48 & 232.04 & 226.55 \\
\hline 0.20 & 179.38 & 167.52 & 163.65 & 172.04 & 168.08 & 167.65 \\
\hline 0.25 & 127.30 & 131.31 & 127.21 & 123.41 & 123.80 & 117.32 \\
\hline 0.30 & 97.51 & 94.79 & 91.32 & 84.01 & 86.92 & 85.30 \\
\hline 0.35 & 73.81 & 68.87 & 66.20 & 62.00 & 60.14 & 55.43 \\
\hline 0.40 & 52.68 & 53.59 & 47.55 & 43.98 & 41.21 & 38.32 \\
\hline 0.45 & 41.22 & 39.59 & 33.94 & 31.65 & 29.71 & 25.57 \\
\hline $\mathbf{0 . 5 0}$ & 29.98 & 31.02 & 23.92 & 23.32 & 19.65 & 19.23 \\
\hline 0.60 & 18.32 & 17.83 & 13.18 & 11.62 & 10.04 & 8.96 \\
\hline 0.70 & 10.91 & 10.21 & 7.27 & 6.31 & 5.14 & 4.84 \\
\hline 0.80 & 7.01 & 6.40 & 4.29 & 3.72 & 3.24 & 2.98 \\
\hline 0.90 & 4.76 & 3.96 & 2.86 & 2.44 & 2.29 & 2.27 \\
\hline \multirow[t]{2}{*}{1.00} & 3.35 & 2.75 & 1.97 & 1.83 & 1.79 & 1.74 \\
\hline & \multicolumn{6}{|c|}{$n=10$} \\
\hline 0.00 & 368.72 & 363.25 & 372.01 & 371.55 & 364.20 & 375.43 \\
\hline 0.10 & 243.56 & 239.42 & 236.08 & 247.27 & 241.64 & 234.47 \\
\hline 0.15 & 166.65 & 166.54 & 159.15 & 156.54 & 156.11 & 156.12 \\
\hline 0.20 & 105.74 & 104.59 & 100.42 & 98.18 & 95.55 & 92.74 \\
\hline 0.25 & 71.44 & 70.18 & 67.41 & 61.50 & 61.18 & 54.54 \\
\hline $\mathbf{0 . 3 0}$ & 48.25 & 44.94 & 40.13 & 37.54 & 35.44 & 32.74 \\
\hline $\mathbf{0 . 3 5}$ & 31.26 & 30.24 & 24.81 & 23.77 & 21.10 & 20.15 \\
\hline 0.40 & 22.14 & 20.90 & 17.17 & 14.34 & 12.82 & 11.26 \\
\hline 0.45 & 15.34 & 13.80 & 11.18 & 9.29 & 7.91 & 6.85 \\
\hline $\mathbf{0 . 5 0}$ & 11.14 & 10.06 & 7.05 & 5.81 & 5.08 & 4.69 \\
\hline $\mathbf{0 . 6 0}$ & 5.93 & 5.11 & 3.38 & 2.91 & 2.67 & 2.63 \\
\hline $\mathbf{0 . 7 0}$ & 3.54 & 2.83 & 2.10 & 1.96 & 1.83 & 1.75 \\
\hline $\begin{array}{l}\mathbf{0 . 8 0} \\
\end{array}$ & 2.28 & 1.84 & 1.51 & 1.45 & 1.45 & 1.35 \\
\hline 0.90 & 1.65 & 1.41 & 1.23 & 1.20 & 1.22 & 1.20 \\
\hline \multirow[t]{2}{*}{1.00} & 1.33 & 1.20 & 1.10 & 1.10 & 1.10 & 1.10 \\
\hline & \multicolumn{6}{|c|}{$\mathbf{n}=\mathbf{2 0}$} \\
\hline 0.00 & 373.20 & 363.07 & 371.71 & 368.37 & 387.85 & 380.42 \\
\hline $\mathbf{0 . 1 0}$ & 187.05 & 178.31 & 164.43 & 162.52 & 169.24 & 165.93 \\
\hline 0.15 & 99.92 & 96.17 & 89.27 & 90.94 & 83.60 & 82.10 \\
\hline 0.20 & 55.23 & 51.52 & 46.89 & 45.13 & 43.16 & 38.57 \\
\hline 0.25 & 30.89 & 30.44 & 24.88 & 22.30 & 19.84 & 17.63 \\
\hline $\mathbf{0 . 3 0}$ & 18.68 & 18.04 & 13.51 & 11.69 & 9.97 & 8.97 \\
\hline $\mathbf{0 . 3 5}$ & 11.50 & 10.01 & 7.68 & 6.19 & 5.35 & 4.78 \\
\hline 0.40 & 7.06 & 6.33 & 4.26 & 3.71 & 3.26 & 3.13 \\
\hline 0.45 & 4.84 & 4.08 & 2.71 & 2.48 & 2.28 & 2.19 \\
\hline $\begin{array}{l}\mathbf{0 . 5 0} \\
\end{array}$ & 3.26 & 2.74 & 1.99 & 1.79 & 1.74 & 1.72 \\
\hline 0.60 & 1.91 & 1.57 & 1.33 & 1.31 & 1.30 & 1.30 \\
\hline 0.70 & 1.35 & 1.22 & 1.11 & 1.11 & 1.12 & 1.14 \\
\hline $\begin{array}{l}0.80 \\
\end{array}$ & 1.13 & 1.09 & 1.04 & 1.04 & 1.05 & 1.04 \\
\hline 0.90 & 1.04 & 1.03 & 1.03 & 1.02 & 1.03 & 1.02 \\
\hline \multirow[t]{3}{*}{1.00} & 1.01 & 1.01 & 1.01 & 1.01 & 1.01 & 1.01 \\
\hline & \multicolumn{6}{|c|}{$\mathbf{n}=\mathbf{3 0}$} \\
\hline & $m=1$ & $\mathrm{~m}=\mathbf{2}$ & $\mathbf{m}=\mathbf{3}$ & $\mathrm{m}=4$ & $m=5$ & $m=6$ \\
\hline 0.00 & 367.57 & 359.89 & 363.66 & 378.56 & 363.03 & 370.48 \\
\hline 0.10 & 134.40 & 135.03 & 128.14 & 126.91 & 125.67 & 122.93 \\
\hline
\end{tabular}




\begin{tabular}{|c|c|c|c|c|c|c|}
\hline 0.15 & 67.38 & 64.10 & 59.63 & 57.32 & 52.66 & 48.22 \\
\hline 0.20 & 32.16 & 30.59 & 26.22 & 23.80 & 21.41 & 20.00 \\
\hline 0.25 & 17.52 & 16.43 & 12.45 & 10.31 & 9.04 & 8.35 \\
\hline 0.30 & 9.62 & 8.91 & 6.22 & 5.08 & 4.47 & 4.10 \\
\hline 0.35 & 5.61 & 4.79 & 3.37 & 2.95 & 2.73 & 2.53 \\
\hline 0.40 & 3.55 & 3.02 & 2.11 & 1.89 & 1.86 & 1.86 \\
\hline 0.45 & 2.40 & 2.03 & 1.65 & 1.44 & 1.49 & 1.44 \\
\hline 0.50 & 1.79 & 1.55 & 1.29 & 1.30 & 1.24 & 1.24 \\
\hline 0.60 & 1.25 & 1.17 & 1.10 & 1.09 & 1.07 & 1.08 \\
\hline 0.70 & 1.06 & 1.05 & 1.02 & 1.03 & 1.02 & 1.02 \\
\hline 0.80 & 1.02 & 1.01 & 1.01 & 1.01 & 1.02 & 1.01 \\
\hline 0.90 & 1.00 & 1.00 & 1.00 & 1.00 & 1.01 & 1.00 \\
\hline 1.00 & 1.00 & 1.00 & 1.00 & 1.00 & 1.00 & 1.00 \\
\hline & \multicolumn{6}{|c|}{$n=40$} \\
\hline 0.00 & 367.22 & 369.10 & 362.10 & 368.51 & 364.33 & 378.94 \\
\hline 0 & 107.78 & 107.32 & 104.35 & 96.82 & 96.58 & 93.51 \\
\hline 0.15 & 47.07 & 45.50 & 41.34 & 35.15 & 36.73 & 32.20 \\
\hline 0.20 & 21.81 & 21.80 & 16.39 & 14.75 & 12.58 & 10.65 \\
\hline 0.25 & 10.66 & 9.67 & 6.91 & 5.90 & 5.24 & 4.60 \\
\hline 0.30 & 6.01 & 5.05 & 3.48 & 2.91 & 2.66 & 2.63 \\
\hline 0.35 & 3.29 & 2.92 & 2.07 & 1.88 & 1.82 & 1.78 \\
\hline 0.40 & 2.27 & 1.87 & 1.48 & 1.41 & 1.41 & 1.38 \\
\hline 0.45 & 1.64 & 1.41 & 1.22 & 1.23 & 1.19 & 1.19 \\
\hline 0.50 & 1.32 & 1.21 & 1.13 & 1.10 & 1.11 & 1.09 \\
\hline 0.60 & 1.10 & 1.04 & 1.04 & 1.03 & 1.04 & 1.03 \\
\hline 0.70 & 1.02 & 1.01 & 1.00 & 1.01 & 1.01 & 1.01 \\
\hline 0.80 & 1.00 & 1.00 & 1.00 & 1.00 & 1.00 & 1.00 \\
\hline 0.90 & 1.00 & 1.00 & 1.00 & 1.00 & 1.00 & 1.00 \\
\hline 1.00 & 1.00 & 1.00 & 1.00 & 1.00 & 1.00 & 1.00 \\
\hline
\end{tabular}

Table 4: $\quad c_{2}=0.67 c_{1}, A R L_{0}=500$

\begin{tabular}{|c|c|c|c|c|c|c|}
\hline & \multicolumn{6}{|c|}{$n=5$} \\
\hline shift & $\mathbf{m}=\mathbf{1}$ & $\mathbf{m}=\mathbf{2}$ & $\mathbf{m}=\mathbf{3}$ & $\mathrm{m}=4$ & $m=5$ & $m=6$ \\
\hline 0.00 & 503.70 & 496.28 & 506.52 & 492.50 & 510.94 & 498.46 \\
\hline 0.10 & 420.16 & 394.05 & 419.74 & 406.53 & 414.17 & 424.20 \\
\hline 0.15 & 315.20 & 312.99 & 327.72 & 309.72 & 331.92 & 333.57 \\
\hline 0.20 & 243.93 & 233.20 & 230.85 & 220.15 & 234.03 & 237.78 \\
\hline 0.25 & 176.50 & 174.39 & 169.09 & 158.96 & 163.11 & 162.71 \\
\hline $\mathbf{0 . 3 0}$ & 125.41 & 120.77 & 115.27 & 109.34 & 110.61 & 102.15 \\
\hline $\mathbf{0 . 3 5}$ & 93.69 & 87.01 & 83.73 & 73.01 & 72.40 & 66.78 \\
\hline 0.40 & 68.53 & 66.96 & 59.02 & 49.95 & 47.19 & 40.11 \\
\hline 0.45 & 51.46 & 48.38 & 42.20 & 32.02 & 29.31 & 25.18 \\
\hline $\mathbf{0 . 5 0}$ & 37.78 & 35.80 & 27.69 & 23.21 & 18.67 & 15.49 \\
\hline $\mathbf{0 . 6 0}$ & 22.36 & 18.38 & 13.27 & 10.28 & 8.03 & 6.58 \\
\hline $\mathbf{0 . 7 0}$ & 12.97 & 10.49 & 6.47 & 4.83 & 3.93 & 3.62 \\
\hline $\begin{array}{l}0.80 \\
\end{array}$ & 7.74 & 5.73 & 3.49 & 2.67 & 2.46 & 2.48 \\
\hline 0.90 & 4.90 & 3.46 & 2.25 & 1.98 & 1.82 & 1.73 \\
\hline 1.00 & 3.35 & 2.31 & 1.60 & 1.46 & 1.44 & 1.42 \\
\hline & \multicolumn{6}{|c|}{$n=10$} \\
\hline 0.00 & 500.05 & 490.53 & 517.38 & 489.23 & 507.31 & 501.60 \\
\hline 0.10 & 338.07 & 329.18 & 326.53 & 322.68 & 344.08 & 352.10 \\
\hline $\mathbf{0 . 1 5}$ & 225.02 & 223.38 & 216.26 & 197.47 & 212.73 & 218.94 \\
\hline 0.20 & 147.33 & 137.26 & 132.19 & 123.03 & 125.72 & 123.84 \\
\hline
\end{tabular}




\begin{tabular}{|c|c|c|c|c|c|c|}
\hline 0.25 & 90.28 & 87.70 & 81.06 & 73.51 & 72.87 & 64.84 \\
\hline $\mathbf{0 . 3 0}$ & 59.28 & 58.18 & 47.99 & 40.62 & 38.69 & 32.28 \\
\hline 0.35 & 39.80 & 35.95 & 28.63 & 23.54 & 19.33 & 16.49 \\
\hline 0.40 & 25.75 & 22.45 & 16.87 & 13.17 & 10.55 & 8.79 \\
\hline 0.45 & 17.24 & 15.12 & 10.41 & 7.41 & 6.31 & 5.14 \\
\hline 0.50 & 11.95 & 9.97 & 6.09 & 4.36 & 4.11 & 3.47 \\
\hline 0.60 & 6.20 & 4.55 & 2.89 & 2.17 & 2.06 & 1.97 \\
\hline 0.70 & 3.43 & 2.37 & 1.60 & 1.50 & 1.44 & 1.45 \\
\hline 0.80 & 2.14 & 1.56 & 1.22 & 1.17 & 1.18 & 1.17 \\
\hline 0.90 & 1.56 & 1.23 & 1.04 & 1.03 & 1.01 & 1.04 \\
\hline 1.00 & 1.27 & 1.09 & 1.00 & 1.00 & 1.00 & 1.00 \\
\hline & \multicolumn{6}{|c|}{$\mathbf{n}=\mathbf{2 0}$} \\
\hline 0.00 & 502.26 & 498.43 & 507.20 & 490.32 & 510.59 & 507.02 \\
\hline 0.10 & 236.94 & 235.45 & 242.09 & 224.44 & 236.24 & 243.79 \\
\hline 0.15 & 134.73 & 124.48 & 118.20 & 107.30 & 108.88 & 102.71 \\
\hline 0.20 & 68.78 & 64.31 & 57.83 & 52.47 & 45.44 & 44.31 \\
\hline 0.25 & 38.92 & 36.39 & 28.22 & 22.57 & 19.37 & 15.82 \\
\hline 0.30 & 21.80 & 19.11 & 13.51 & 10.09 & 8.00 & 6.33 \\
\hline 0.35 & 12.88 & 10.47 & 6.32 & 4.66 & 4.16 & 3.47 \\
\hline 0.40 & 8.08 & 5.67 & 3.62 & 2.63 & 2.53 & 2.41 \\
\hline 0.45 & 4.80 & 3.34 & 2.18 & 1.97 & 1.83 & 1.79 \\
\hline 0.50 & 3.41 & 2.33 & 1.56 & 1.45 & 1.42 & 1.43 \\
\hline 0.60 & 1.84 & 1.39 & 1.14 & 1.10 & 1.10 & 1.07 \\
\hline 0.70 & 1.31 & 1.08 & 1.01 & 1.00 & 1.00 & 1.00 \\
\hline 0.80 & 1.10 & 1.03 & 1.00 & 1.00 & 1.00 & 1.00 \\
\hline 0.90 & 1.04 & 1.01 & 0.99 & 1.00 & 1.00 & 1.00 \\
\hline \multirow[t]{3}{*}{1.00} & 1.01 & 1.00 & 1.00 & 1.00 & 1.00 & 1.00 \\
\hline & \multicolumn{6}{|c|}{$\mathbf{n}=\mathbf{3 0}$} \\
\hline & $\mathbf{m}=\mathbf{1}$ & $\mathbf{m}=\mathbf{2}$ & $\mathbf{m}=\mathbf{3}$ & $\mathrm{m}=4$ & $\mathrm{~m}=5$ & $\mathrm{~m}=6$ \\
\hline 0.00 & 518.33 & 493.85 & 509.89 & 494.63 & 510.44 & 495.79 \\
\hline 0.10 & 187.60 & 175.50 & 175.78 & 160.62 & 161.77 & 165.72 \\
\hline 0.15 & 83.38 & 83.74 & 72.82 & 65.08 & 60.25 & 57.72 \\
\hline 0.20 & 40.19 & 38.14 & 29.93 & 23.78 & 22.15 & 17.87 \\
\hline 0.25 & 20.67 & 17.55 & 12.13 & 8.82 & 6.81 & 5.77 \\
\hline 0.30 & 10.69 & 8.29 & 5.12 & 3.79 & 3.31 & 3.06 \\
\hline 0.35 & 5.95 & 4.39 & 2.58 & 2.25 & 1.96 & 1.99 \\
\hline 0.40 & 3.53 & 2.42 & 1.71 & 1.52 & 1.53 & 1.49 \\
\hline 0.45 & 2.37 & 1.70 & 1.25 & 1.24 & 1.24 & 1.19 \\
\hline 0.50 & 1.75 & 1.31 & 1.10 & 1.08 & 1.04 & 1.06 \\
\hline 0.60 & 1.21 & 1.05 & 1.00 & 1.00 & 1.00 & 1.00 \\
\hline 0.70 & 1.06 & 1.02 & 1.00 & 1.00 & 1.00 & 1.00 \\
\hline 0.80 & 1.01 & 1.00 & 1.00 & 1.00 & 1.00 & 1.00 \\
\hline 0.90 & 1.00 & 1.00 & 1.00 & 1.00 & 1.00 & 1.00 \\
\hline \multirow[t]{2}{*}{1.00} & 1.00 & 1.00 & 1.00 & 1.00 & 1.00 & 1.00 \\
\hline & \multicolumn{6}{|c|}{$n=40$} \\
\hline 0.00 & 518.74 & 492.11 & 515.11 & 485.51 & 501.88 & 502.61 \\
\hline 0.10 & 148.84 & 141.49 & 131.61 & 121.63 & 125.62 & 114.94 \\
\hline 0.15 & 59.29 & 55.74 & 48.96 & 41.08 & 39.45 & 34.00 \\
\hline 0.20 & 25.42 & 23.68 & 17.26 & 12.82 & 10.49 & 9.36 \\
\hline 0.25 & 11.87 & 10.15 & 6.03 & 4.59 & 3.60 & 3.46 \\
\hline 0.30 & 6.24 & 4.39 & 2.80 & 2.21 & 2.07 & 2.07 \\
\hline 0.35 & 3.33 & 2.36 & 1.71 & 1.48 & 1.47 & 1.44 \\
\hline 0.40 & 2.23 & 1.54 & 1.23 & 1.16 & 1.18 & 1.15 \\
\hline 0.45 & 1.58 & 1.19 & 1.04 & 1.04 & 1.03 & 1.05 \\
\hline 0.50 & 1.27 & 1.07 & 1.00 & 1.00 & 1.00 & 1.00 \\
\hline
\end{tabular}




\begin{tabular}{|l|l|l|l|l|r|r|}
\hline $\mathbf{0 . 6 0}$ & 1.06 & 1.02 & 1.00 & 1.00 & 1.00 & 1.00 \\
\hline $\mathbf{0 . 7 0}$ & 1.01 & 1.00 & 1.00 & 1.00 & 1.00 & 1.00 \\
\hline $\mathbf{0 . 8 0}$ & 1.00 & 1.00 & 1.00 & 1.00 & 1.00 & 1.00 \\
\hline $\mathbf{0 . 9 0}$ & 1.00 & 1.00 & 1.00 & 1.00 & 1.00 & 1.00 \\
\hline $\mathbf{1 . 0 0}$ & 1.00 & 1.00 & 1.00 & 1.00 & 1.00 & 1.00 \\
\hline
\end{tabular}

Table 5: $\quad c_{2}=0.675 c_{1}, A R L_{0}=370$

\begin{tabular}{|c|c|c|c|c|c|c|}
\hline & \multicolumn{6}{|c|}{$n=5$} \\
\hline shift & $\mathrm{m}=1$ & $\mathrm{~m}=\mathbf{2}$ & $\mathbf{m}=\mathbf{3}$ & $\mathrm{m}=4$ & $m=5$ & $m=6$ \\
\hline 0.00 & 375.18 & 366.43 & 373.63 & 374.88 & 365.23 & 385.27 \\
\hline 0.10 & 298.61 & 299.11 & 305.49 & 294.76 & 283.47 & 300.40 \\
\hline 0.15 & 232.63 & 233.46 & 233.97 & 228.04 & 215.34 & 225.36 \\
\hline 0.20 & 171.86 & 176.81 & 169.65 & 163.28 & 154.87 & 158.36 \\
\hline 0.25 & 134.31 & 127.50 & 120.54 & 114.99 & 108.72 & 104.51 \\
\hline 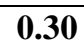 & 98.37 & 94.94 & 85.98 & 79.42 & 75.80 & 71.40 \\
\hline 0.35 & 70.11 & 69.53 & 64.31 & 56.63 & 49.02 & 46.64 \\
\hline 0.40 & 51.31 & 51.08 & 42.61 & 37.15 & 32.51 & 28.84 \\
\hline 0.45 & 38.28 & 37.73 & 29.57 & 25.06 & 20.34 & 17.43 \\
\hline 0.50 & 29.24 & 27.19 & 21.08 & 17.11 & 13.46 & 10.85 \\
\hline 0.60 & 17.65 & 15.16 & 10.36 & 8.02 & 6.23 & 5.14 \\
\hline $\mathbf{0 . 7 0}$ & 10.61 & 8.21 & 5.18 & 3.91 & 3.36 & 3.13 \\
\hline 0.80 & 6.47 & 4.92 & 2.91 & 2.40 & 2.31 & 2.17 \\
\hline 0.90 & 4.22 & 3.00 & 1.99 & 1.73 & 1.70 & 1.64 \\
\hline 1.00 & 2.92 & 2.08 & 1.47 & 1.39 & 1.36 & 1.35 \\
\hline & \multicolumn{6}{|c|}{$n=10$} \\
\hline 0.00 & 373.61 & 374.78 & 375.84 & 377.04 & 379.51 & 384.82 \\
\hline 0.10 & 246.31 & 242.02 & 245.50 & 231.66 & 237.65 & 236.53 \\
\hline 0.15 & 163.33 & 163.69 & 166.61 & 151.89 & 152.32 & 140.21 \\
\hline 0.20 & 105.60 & 104.96 & 99.70 & 89.20 & 85.28 & 79.29 \\
\hline 0.25 & 67.91 & 69.60 & 58.83 & 55.22 & 49.31 & 43.74 \\
\hline $\mathbf{0 . 3 0}$ & 46.12 & 42.57 & 36.03 & 29.86 & 26.14 & 21.74 \\
\hline 0.35 & 30.09 & 28.47 & 21.43 & 16.76 & 14.28 & 10.77 \\
\hline 0.40 & 20.74 & 18.40 & 13.41 & 9.64 & 8.02 & 6.57 \\
\hline 0.45 & 14.43 & 12.51 & 7.86 & 5.60 & 4.82 & 4.22 \\
\hline 0.50 & 10.29 & 7.99 & 5.21 & 3.83 & 3.16 & 3.02 \\
\hline 0.60 & 5.30 & 3.77 & 2.42 & 2.08 & 1.95 & 1.87 \\
\hline 0.70 & 3.04 & 2.25 & 1.50 & 1.43 & 1.39 & 1.41 \\
\hline 0.80 & 1.99 & 1.50 & 1.18 & 1.17 & 1.13 & 1.14 \\
\hline 0.90 & 1.51 & 1.20 & 1.04 & 1.02 & 1.04 & 1.03 \\
\hline 1.00 & 1.24 & 1.08 & 1.00 & 1.00 & 1.00 & 1.00 \\
\hline & \multicolumn{6}{|c|}{$\mathrm{n}=\mathbf{2 0}$} \\
\hline $\begin{array}{l}0.00 \\
\end{array}$ & 371.82 & 378.41 & 383.60 & 367.01 & 365.81 & 382.51 \\
\hline 0.10 & 178.82 & 178.62 & 168.16 & 170.47 & 160.64 & 157.51 \\
\hline 0.15 & 92.78 & 96.53 & 86.31 & 80.89 & 70.85 & 70.29 \\
\hline 0.20 & 53.37 & 50.82 & 43.90 & 39.62 & 31.64 & 28.84 \\
\hline 0.25 & 30.60 & 26.30 & 20.68 & 16.61 & 14.02 & 11.09 \\
\hline $\begin{array}{l}\mathbf{0 . 3 0} \\
\end{array}$ & 17.31 & 15.19 & 10.07 & 7.35 & 6.00 & 5.33 \\
\hline 0.35 & 10.43 & 8.37 & 4.99 & 3.95 & 3.53 & 3.29 \\
\hline 0 & 6.46 & 5.03 & 3.00 & 2.41 & 2.34 & 2.16 \\
\hline 0.45 & 4.10 & 3.10 & 2.00 & 1.80 & 1.72 & 1.67 \\
\hline $\begin{array}{l}\mathbf{0 . 5 0} \\
\end{array}$ & 2.96 & 2.07 & 1.49 & 1.41 & 1.37 & 1.37 \\
\hline 0 & 1.65 & 1.36 & 1.08 & 1.08 & 1.05 & 1.07 \\
\hline $\begin{array}{l}\mathbf{0 . 7 0} \\
\end{array}$ & 1.26 & 1.11 & 1.00 & 1.00 & 1.00 & 1.00 \\
\hline
\end{tabular}




\begin{tabular}{|c|c|c|c|c|c|c|}
\hline 0.80 & 1.08 & 1.02 & 1.00 & 1.00 & 1.00 & 1.00 \\
\hline 0.90 & 1.03 & 1.01 & 1.00 & 1.00 & 1.00 & 1.00 \\
\hline 1.00 & 1.01 & 1.00 & 1.00 & 1.00 & 1.00 & 1.00 \\
\hline & \multicolumn{6}{|c|}{$\mathbf{n}=\mathbf{3 0}$} \\
\hline & $m=1$ & $m=2$ & $\mathbf{m}=\mathbf{3}$ & $\mathrm{m}=4$ & $m=5$ & $m=6$ \\
\hline 0.00 & 370.62 & 366.87 & 383.07 & 374.18 & 369.39 & 384.21 \\
\hline 0.10 & 132.15 & 130.37 & 126.45 & 124.01 & 110.46 & 113.27 \\
\hline 0.15 & 63.67 & 59.19 & 54.29 & 48.33 & 43.29 & 39.23 \\
\hline 0.20 & 33.60 & 28.50 & 21.97 & 18.61 & 14.82 & 12.07 \\
\hline 0.25 & 16.14 & 13.57 & 9.80 & 6.85 & 5.68 & 4.68 \\
\hline 0.30 & 8.81 & 6.76 & 4.32 & 3.38 & 2.82 & 2.78 \\
\hline 0.35 & 5.04 & 3.56 & 2.37 & 1.93 & 1.87 & 1.83 \\
\hline 0.40 & 3.19 & 2.20 & 1.56 & 1.46 & 1.42 & 1.36 \\
\hline 0.45 & 2.11 & 1.54 & 1.23 & 1.19 & 1.16 & 1.15 \\
\hline 0.50 & 1.60 & 1.22 & 1.09 & 1.09 & 1.05 & 1.05 \\
\hline 0.60 & 1.17 & 1.06 & 1.00 & 0.99 & 1.00 & 1.00 \\
\hline 0.70 & 1.04 & 0.99 & 1.00 & 1.00 & 1.01 & 1.00 \\
\hline 0.80 & 1.01 & 1.01 & 1.00 & 1.00 & 1.00 & 1.00 \\
\hline 0.90 & 1.00 & 1.00 & 1.00 & 1.00 & 1.00 & 1.00 \\
\hline \multirow[t]{2}{*}{1.00} & 1.00 & 1.00 & 1.00 & 1.00 & 1.00 & 1.00 \\
\hline & \multicolumn{6}{|c|}{$n=40$} \\
\hline 0.00 & 368.27 & 375.31 & 375.27 & 362.73 & 360.38 & 375.89 \\
\hline 0.10 & 110.47 & 104.51 & 94.94 & 93.23 & 85.92 & 80.58 \\
\hline 0.15 & 46.09 & 43.67 & 36.84 & 31.04 & 27.10 & 21.58 \\
\hline 0.20 & 20.54 & 18.58 & 13.47 & 9.55 & 7.47 & 6.79 \\
\hline 0.25 & 10.29 & 7.81 & 4.86 & 3.86 & 3.20 & 3.17 \\
\hline 0.30 & 5.06 & 4.04 & 2.60 & 2.03 & 1.94 & 1.91 \\
\hline 0.35 & 2.97 & 2.10 & 1.55 & 1.45 & 1.38 & 1.35 \\
\hline 0.40 & 2.01 & 1.51 & 1.22 & 1.13 & 1.13 & 1.12 \\
\hline 0.45 & 1.46 & 1.18 & 1.05 & 1.04 & 1.04 & 1.00 \\
\hline 0.50 & 1.22 & 1.08 & 1.01 & 1.02 & 1.01 & 1.01 \\
\hline 0.60 & 1.03 & 1.01 & 1.00 & 1.00 & 1.00 & 1.00 \\
\hline 0.70 & 1.01 & 1.00 & 1.00 & 1.00 & 1.00 & 1.00 \\
\hline 0.80 & 1.00 & 1.00 & 1.00 & 1.00 & 1.00 & 1.00 \\
\hline 0.90 & 1.00 & 1.00 & 1.00 & 1.00 & 1.00 & 1.00 \\
\hline 1.00 & 1.00 & 1.00 & 1.00 & 1.00 & 1.00 & 1.00 \\
\hline
\end{tabular}

Table 6: $\quad \mathrm{c}_{2}=0.50 \mathrm{c}_{1}, \mathrm{ARL}_{0}=370$

\begin{tabular}{|l|r|r|r|r|r|}
\hline & \multicolumn{5}{|c|}{$\mathbf{n = 5}$} \\
\hline Shift & $\mathbf{m}=\mathbf{1}$ & $\mathbf{m}=\mathbf{2}$ & $\mathbf{m = 3}$ & $\mathbf{m}=\mathbf{4}$ & \multicolumn{1}{|c|}{$\mathbf{m}=\mathbf{5}$} \\
\hline $\mathbf{0 . 0 0}$ & 373.52 & 366.40 & 370.70 & 362.81 & 376.58 \\
\hline $\mathbf{0 . 1 0}$ & 308.64 & 286.88 & 305.03 & 285.78 & 328.06 \\
\hline $\mathbf{0 . 1 5}$ & 239.01 & 215.20 & 212.29 & 210.67 & 226.50 \\
\hline $\mathbf{0 . 2 0}$ & 178.04 & 159.86 & 155.09 & 146.47 & 161.16 \\
\hline $\mathbf{0 . 2 5}$ & 129.01 & 117.35 & 103.98 & 85.47 & 92.75 \\
\hline $\mathbf{0 . 3 0}$ & 91.11 & 82.60 & 63.34 & 50.10 & 42.49 \\
\hline $\mathbf{0 . 3 5}$ & 67.37 & 55.88 & 42.24 & 27.73 & 19.40 \\
\hline $\mathbf{0 . 4 0}$ & 49.50 & 39.33 & 25.12 & 13.51 & 9.57 \\
\hline $\mathbf{0 . 4 5}$ & 35.42 & 25.75 & 14.47 & 7.60 & 5.87 \\
\hline $\mathbf{0 . 5 0}$ & 24.89 & 17.28 & 8.14 & 4.34 & 3.85 \\
\hline $\mathbf{0 . 6 0}$ & 14.36 & 7.54 & 3.26 & 2.50 & 2.24 \\
\hline $\mathbf{0 . 7 0}$ & 7.84 & 3.55 & 1.92 & 1.66 & 1.60 \\
\hline $\mathbf{0 . 8 0}$ & 4.77 & 2.06 & 1.33 & 1.17 & 1.20 \\
\hline
\end{tabular}




\begin{tabular}{|c|c|c|c|c|c|}
\hline 0.90 & 3.06 & 1.39 & 1.00 & 1.00 & 1.00 \\
\hline 1.00 & 2.17 & 1.09 & 1.00 & 1.00 & 1.00 \\
\hline & \multicolumn{5}{|c|}{$\mathrm{n}=10$} \\
\hline 0.00 & 372.38 & 373.12 & 372.50 & 361.53 & 361.68 \\
\hline 0.10 & 254.02 & 235.45 & 233.46 & 220.75 & 254.71 \\
\hline 0.15 & 168.07 & 152.30 & 141.07 & 126.73 & 133.28 \\
\hline 0.20 & 102.81 & 92.08 & 78.85 & 60.55 & 54.18 \\
\hline 0.25 & 64.47 & 56.01 & 39.44 & 22.65 & 19.16 \\
\hline 0.30 & 41.68 & 32.28 & 18.21 & 9.93 & 7.26 \\
\hline 0.35 & 25.28 & 17.85 & 8.64 & 4.93 & 3.98 \\
\hline 0.40 & 17.80 & 10.82 & 4.24 & 2.97 & 2.62 \\
\hline 0.45 & 11.46 & 5.97 & 2.68 & 1.99 & 1.99 \\
\hline 0.50 & 8.04 & 3.42 & 1.82 & 1.53 & 1.57 \\
\hline 0.60 & 3.94 & 1.69 & 1.17 & 1.03 & 1.05 \\
\hline 0.70 & 2.25 & 1.11 & 1.00 & 1.00 & 1.00 \\
\hline $\mathbf{0 . 8 0}$ & 1.52 & 1.00 & 1.00 & 1.00 & 1.00 \\
\hline 0.90 & 1.22 & 1.00 & 1.00 & 1.00 & 1.00 \\
\hline \multirow[t]{2}{*}{1.00} & 1.08 & 1.00 & 1.00 & 1.00 & 1.00 \\
\hline & \multicolumn{5}{|c|}{$\mathrm{n}=20$} \\
\hline 0.00 & 377.45 & 364.30 & 365.45 & 367.96 & 364.53 \\
\hline 0.10 & 171.58 & 166.81 & 149.65 & 144.42 & 155.04 \\
\hline 0.15 & 92.20 & 78.24 & 66.18 & 45.82 & 44.69 \\
\hline 0.20 & 48.47 & 39.05 & 24.43 & 12.60 & 9.70 \\
\hline 0.25 & 26.29 & 17.34 & 8.41 & 4.56 & 3.85 \\
\hline 0.30 & 14.61 & 7.32 & 3.33 & 2.45 & 2.37 \\
\hline 0.35 & 8.26 & 3.64 & 2.00 & 1.60 & 1.47 \\
\hline 0.40 & 4.91 & 2.13 & 1.35 & 1.15 & 1.18 \\
\hline 0.45 & 3.03 & 1.42 & 1.01 & 1.00 & 1.00 \\
\hline $\begin{array}{l}\mathbf{0 . 5 0} \\
\end{array}$ & 2.13 & 1.12 & 1.00 & 1.00 & 1.00 \\
\hline 0.60 & 1.34 & 1.00 & 1.00 & 1.00 & 1.00 \\
\hline 0.70 & 1.10 & 1.00 & 1.00 & 1.00 & 1.00 \\
\hline $\mathbf{0 . 8 0}$ & 1.03 & 1.00 & 1.00 & 1.00 & 1.00 \\
\hline 0.90 & 1.01 & 1.00 & 1.00 & 1.00 & 1.00 \\
\hline \multirow[t]{3}{*}{1.00} & 1.00 & 1.00 & 1.00 & 1.00 & 1.00 \\
\hline & \multicolumn{5}{|c|}{$n=30$} \\
\hline & $\mathbf{m}=\mathbf{1}$ & $\mathbf{m}=\mathbf{2}$ & $\mathbf{m}=\mathbf{3}$ & $\mathrm{m}=4$ & $m=5$ \\
\hline 0.00 & 376.69 & 376.24 & 377.20 & 365.78 & 365.00 \\
\hline 0.10 & 128.87 & 119.92 & 110.61 & 95.31 & 94.06 \\
\hline 0.15 & 59.62 & 51.14 & 35.41 & 20.21 & 15.00 \\
\hline 0.20 & 26.65 & 19.84 & 9.92 & 5.13 & 4.42 \\
\hline 0.25 & 13.31 & 6.93 & 3.05 & 2.26 & 2.22 \\
\hline \begin{tabular}{|c|}
0.30 \\
\end{tabular} & 6.56 & 3.00 & 1.66 & 1.40 & 1.43 \\
\hline 0.35 & 3.71 & 1.74 & 1.09 & 1.04 & 1.07 \\
\hline 0.40 & 2.31 & 1.17 & 1.00 & 1.00 & 1.00 \\
\hline 0.45 & 1.61 & 1.00 & 1.00 & 1.00 & 1.00 \\
\hline $\mathbf{0 . 5 0}$ & 1.30 & 1.00 & 1.00 & 1.00 & 1.00 \\
\hline $\mathbf{0 . 6 0}$ & 1.06 & 1.00 & 1.00 & 1.00 & 1.00 \\
\hline 0.70 & 1.01 & 1.00 & 1.00 & 1.00 & 1.00 \\
\hline 0.80 & 1.00 & 1.00 & 1.00 & 1.00 & 1.00 \\
\hline 0.90 & 1.00 & 1.00 & 1.00 & 1.00 & 1.00 \\
\hline \multirow[t]{2}{*}{1.00} & 1.00 & 1.00 & 1.00 & 1.00 & 1.00 \\
\hline & \multicolumn{5}{|c|}{$n=40$} \\
\hline 0.00 & 364.31 & 369.90 & 377.45 & 365.94 & 378.34 \\
\hline 0.10 & 103.98 & 92.03 & 74.81 & 62.46 & 56.32 \\
\hline 0.15 & 42.12 & 32.72 & 18.95 & 9.95 & 7.40 \\
\hline
\end{tabular}




\begin{tabular}{|l|r|r|r|r|r|}
\hline $\mathbf{0 . 2 0}$ & 17.42 & 10.44 & 4.48 & 2.84 & 2.75 \\
\hline $\mathbf{0 . 2 5}$ & 7.94 & 3.43 & 1.87 & 1.65 & 1.62 \\
\hline $\mathbf{0 . 3 0}$ & 3.95 & 1.74 & 1.14 & 1.09 & 1.06 \\
\hline $\mathbf{0 . 3 5}$ & 2.23 & 1.07 & 1.00 & 1.00 & 1.00 \\
\hline $\mathbf{0 . 4 0}$ & 1.53 & 1.00 & 1.00 & 1.00 & 1.00 \\
\hline $\mathbf{0 . 4 5}$ & 1.22 & 1.00 & 1.00 & 1.00 & 1.00 \\
\hline $\mathbf{0 . 5 0}$ & 1.10 & 1.00 & 1.00 & 1.00 & 1.00 \\
\hline $\mathbf{0 . 6 0}$ & 1.01 & 1.00 & 1.00 & 1.00 & 1.00 \\
\hline $\mathbf{0 . 7 0}$ & 1.00 & 1.00 & 1.00 & 1.00 & 1.00 \\
\hline $\mathbf{0 . 8 0}$ & 1.00 & 1.00 & 1.00 & 1.00 & 1.00 \\
\hline $\mathbf{0 . 9 0}$ & 1.00 & 1.00 & 1.00 & 1.00 & 1.00 \\
\hline $\mathbf{1 . 0 0}$ & 1.00 & 1.00 & 1.00 & 1.00 & 1.00 \\
\hline
\end{tabular}

Table 7: Summary and Comparison

\begin{tabular}{|c|c|c|c|c|c|c|c|c|}
\hline & Ratio & \multicolumn{7}{|c|}{ Table 2} \\
\hline n & $\mathbf{c}_{2} / \mathbf{c}_{1}$ & Shift & $\mathbf{m}=1$ & $\mathbf{m}=\mathbf{2}$ & $\mathbf{m}=\mathbf{3}$ & $m=4$ & $m=5$ & $m=6$ \\
\hline 5 & 0.75 & 0.80 & 8.76 & 7.62 & 5.10 & 4.46 & 3.73 & 3.34 \\
\hline 10 & 0.75 & 0.60 & 6.98 & 5.96 & 4.16 & 3.53 & 3.10 & 3.02 \\
\hline 20 & 0.75 & 0.40 & 8.71 & 7.36 & 5.28 & 4.28 & 3.78 & 3.48 \\
\hline 30 & 0.75 & 0.35 & 6.71 & 5.64 & 3.90 & 3.31 & 3.03 & 2.81 \\
\hline \multirow[t]{2}{*}{40} & 0.75 & 0.30 & 6.98 & 6.12 & 4.05 & 3.40 & 3.30 & 3.00 \\
\hline & & \multicolumn{7}{|c|}{ Table 3} \\
\hline 5 & 0.75 & 0.80 & 7.01 & 6.40 & 4.29 & 3.72 & 3.24 & 2.98 \\
\hline 10 & 0.75 & 0.60 & 5.93 & 5.11 & 3.38 & 2.91 & 2.67 & 2.63 \\
\hline 20 & 0.75 & 0.40 & 7.06 & 6.33 & 4.26 & 3.71 & 3.26 & 3.13 \\
\hline 30 & 0.75 & 0.30 & 9.62 & 8.91 & 6.22 & 5.08 & 4.47 & 4.10 \\
\hline \multirow[t]{2}{*}{40} & 0.75 & 0.30 & 6.01 & 5.05 & 3.48 & 2.91 & 2.66 & 2.63 \\
\hline & & \multicolumn{7}{|c|}{ Table 4} \\
\hline 5 & 0.67 & $\mathbf{0 . 8 0}$ & 7.74 & 5.73 & 3.49 & 2.67 & 2.46 & 2.48 \\
\hline 10 & 0.67 & 0.60 & 6.20 & 4.55 & 2.89 & 2.17 & 2.06 & 1.97 \\
\hline 20 & 0.67 & 0.40 & 8.08 & 5.67 & 3.62 & 2.63 & 2.53 & 2.41 \\
\hline 30 & 0.67 & $\mathbf{0 . 3 5}$ & 5.95 & 4.39 & 2.58 & 2.25 & 1.96 & 1.99 \\
\hline \multirow[t]{2}{*}{40} & 0.67 & $\mathbf{0 . 3 0}$ & 6.24 & 4.39 & 2.80 & 2.21 & 2.07 & 2.07 \\
\hline & & \multicolumn{7}{|c|}{ Table 5} \\
\hline 5 & 0.67 & 0.80 & 6.47 & 4.92 & 2.91 & 2.40 & 2.31 & 2.17 \\
\hline 10 & 0.67 & 0.50 & 10.29 & 7.99 & 5.21 & 3.83 & 3.16 & 3.02 \\
\hline 20 & 0.67 & 0.35 & 10.43 & 8.37 & 4.99 & 3.95 & 3.53 & 3.29 \\
\hline 30 & 0.67 & $\mathbf{0 . 3 0}$ & 8.81 & 6.76 & 4.32 & 3.38 & 2.82 & 2.78 \\
\hline \multirow[t]{2}{*}{40} & 0.67 & 0.25 & 10.29 & 7.81 & 4.86 & 3.86 & 3.20 & 3.17 \\
\hline & & \multicolumn{7}{|c|}{ Table 6} \\
\hline 5 & 0.50 & 0.70 & 7.84 & 3.55 & 1.92 & 1.66 & 1.60 & N/A \\
\hline 10 & 0.50 & 0.50 & 8.04 & 3.42 & 1.82 & 1.53 & 1.57 & N/A \\
\hline 20 & 0.50 & $\mathbf{0 . 3 5}$ & 8.26 & 3.64 & 2.00 & 1.60 & 1.47 & N/A \\
\hline 30 & 0.50 & 0.30 & 6.56 & 3.00 & 1.66 & 1.40 & 1.43 & N/A \\
\hline 40 & 0.50 & 0.25 & 7.94 & 3.43 & 1.87 & 1.65 & 1.62 & N/A \\
\hline
\end{tabular}




\section{Discussion on Table 7}

The purpose behind the Table 8 is to summarize the results from Tables 2 to 6 so that advantage of proposed extension in repetitive procedure is elaborated more clearly. In the above table lenient and moderate repetitive conditions have been discussed where $c_{2} / c_{1}$ ratios are $0.75,0.67$ and 0.50 . Moreover, it is very easy for the reader to understand the results from the pertinent. Here, $\mathrm{m}=1$ indicates the $\mathrm{ARL}_{1} \mathrm{~s}$ from the existing repetitive sampling and shifts included in the table are those that are detected within $A R L_{1}=10$ in case of existing repetitive criteria. For instance, so far as the results of Table 2 above are concerned, under $n=5$ existing repetitive sampling based control chart detects a smallest shift 0.80 at $A R L_{1}=9$. If the proposed criteria are applied, this shift can be detectable at $\mathrm{ARL}_{1}=8$ with soft restrictions, at $\mathrm{ARL}_{1}=4$ with moderate restrictions and with strict restrictions our control chart can alarm at $A R L_{1}=3$. In another case from the results of Table 6 a shift of 0.25 is alarmed at $A R L_{1}=8$ in existing repetitive case whereas we are able to detect this shift at $A R L_{1}=2$ by applying restricted repetitive sampling. This is evident very clearly that with the proposed schematic existing repetitive sampling based control chart become three to four times efficient in detecting the shifts earlier as well as capable of detecting even smaller shifts at shorter ARL. As we observe that increase in sample size improves the capability of chart to detect smaller shifts even in existing repetitive cases, this property also prevails in restricted repetitive sampling. To observe that how smaller shifts in this proposed restricted sampling based control chart can be detectable, one needs to review the main tables.

\section{Concluding Remarks}

In this article the existing repetitive sampling has been classified to three conditions in terms of ratios between constants $c_{2}$ and $c_{1}$. The criteria behind this classification are lying in Table 1. To apply the proposed criteria only few ratios from three categories have been selected. The researchers have a lot of opportunity to experiment this technique on other possible ratios and moreover in this article mean chart has been used whereas this can be applied to many other charts in variable and attribute areas.

\section{Acknowledgements}

The authors are deeply thankful to editor and reviewers for their valuable suggestions to improve the quality of paper. The work by Muhammad Aslam was supported by fund of the Deanship of Scientific Research (DSR), King Abdulaziz University, Jeddah. The author, Muhammad Aslam, therefore, acknowledge with thanks DSR technical and financial support.

\section{References}

1. Ahmad, L., Aslam, M., \& Jun, C. (2014a). Coal Quality Monitoring With Improved Control Charts. European Journal of Scientific Research, 125(2), 427434.

2. Ahmad, L., Aslam, M., \& Jun, C. (2014b). Designing of X-bar control charts based on process capability index using repetitive sampling. Transactions of the Institute of Measurement and Control, 36(3), 367-374. 
3. Aslam, M., Ahmad, M., \& Osama, H. (2015). Improved Attribute Control Chart for Monitoring Non- Conforming Proportion in High Quality Process. MITTEILUNGEN KLOSTERNEUBURG, 65(4), 300-309.

4. Aslam, M., Azam, M., \& Jun, C. (2014a). A new exponentially weighted moving average sign chart using repetitive sampling. Journal of Process Control, 24(7), 1149-1153.

5. Aslam, M., Azam, M., \& Jun, C. (2016). A Control Chart for COM - Poisson Distribution Using Resampling and Exponentially Weighted. Quality and Reliability Engineering International, 32(March 2015), 727-735.

6. Aslam, M., Azam, M., \& Jun, C.-H. (2014b). New Attributes and Variables Control Charts under Repetitive Sampling. Industrial Engineering \& Management System, 13(1), 101-106.

7. Aslam, M., Azam, M., Khan, N., \& Jun, C. (2015). A mixed control chart to monitor the process. International Journal of Production Research, 53(15), 46834693. http://doi.org/10.1080/00207543.2015.1031354

8. Aslam, M., Jun, C., Fernández, A. J., Ahmad, M., \& Rasool, M. (2014). Repetitive Group Sampling Plan Based on Truncated Tests for Weibull Models. Research Journal of Applied Sciences, Engineering and Technology, 7(10), 19171924.

9. Aslam, M., Khan, N., \& Azam, M. (2016). X-Bar Control Charts for NonNormal Correlated Data Under Repetitive Sampling. Journal of Testing and Evaluation, 44(4), 1-12.

10. Aslam, M., Khan, N., Azam, M., \& Jun, C. (2014). Designing of a new monitoring t-chart using repetitive sampling. Information Sciences, 269, 210-216.

11. Azam, M., Aslam, M., \& Jun, C. (2015). Designing of a hybrid exponentially weighted moving average control chart using repetitive sampling. The International Journal of Advanced Manufacturing Technology, 77, 1927-1933.

12. Balamurali, S., Aslam, M., \& Jun, C. (2015). Testing and Evaluation Economic Design of SkSP-R Skip-Lot Sampling Plan, 43(5). http://doi.org/10.1520/JTE20140081.

13. Lee, H., Aslam, M., Shakeel, Q., Lee, W., \& Jun, C.-H. (2015). A control chart using an auxiliary variable and repetitive sampling for monitoring process mean. Journal of Statistical Computation and Simulation, 85(16), 3289-3296.

14. Sherman, R. E.(1965). Design and evaluation of a repetitive group sampling plan.Technometrics, 7(1), 11-21

15. Wu, C., Aslam, M., Chen, J. C., \& Jun, C. (2015). A repetitive group sampling plan by variables inspection for product acceptance determination. European Journal of Industrial Engineering, 9(3), 308-326.

16. Yen, C., Chang, C., \& Aslam, M. (2015). Repetitive variable acceptance sampling plan for one-sided specification. Journal of Statistical Computation and Simulation, 85(6), 1102-1116. 